\title{
MAPEAMENTO HIDROGEOLÓGICO TEMPORAL DA FORMAÇÃO ALTER DO CHÃO UTILIZANDO SONDAGENS ELÉTRICAS VERTICAIS E CAMINHAMENTO ELÉTRICO NO CAMPUS TAPAJÓS DA UFOPA, SANTARÉM-PA.
}

\author{
Souza L. G. ${ }^{*}$, Barros A. C. S. P. C., Sousa Junior R. O., Rufino, C. S., Silva H. T. A., Alunos do curso de Graduação em
} Geofísica do IEG/UFOPA, Siqueira Neto, A.C., Prof. do curso de Geofísica do IEG/UFOPA

Copyright 2018, SBGf - Sociedade Brasileira de Geofísica

Este texto foi preparado para a apresentação no VIII Simpósio Brasileiro de Geofísica, Salinópolis, 18 a 20 de setembro de 2018. Seu conteúdo foi revisado pelo Comitê Técnico do VIII SimBGf, mas não necessariamente representa a opinião da SBGf ou de seus associados. É proibida a reprodução total ou parcial deste material para propósitos comerciais sem prévia autorização da SBGf.

\section{Resumo}

In all civil engineering works it's important to know the position of the water table, as well as its variations due to climatic agents. This concern is due to the need to know the soil where structures will be fixed, avoiding future problems with the constructions. The contribution of geophysics in geotechnics to hydrogeological research is highlighted by the use of the electrorresistivity method, due to its effectiveness and indirect application. The present work was developed with the collaboration of the Geoelectric Methods Laboratory (LAMGEO) and the Tutorial Education Program (PET / IEG). The main objective of this study was to map the variations in the depth of the groundwater table using Vertical Electrical Survey (VES) on an unpaved road to UFOPA - Campus Tapajós. With the purpose of understanding the oscillation of the level of the saturated zone and the possible relation with the floods of the studied area and, consequently, contribute with information that help in the development of the projects of expansion of the physical infrastructure of the university.

\section{Introdução}

A Universidade Federal do Oeste do Pará, com pouco mais de 08 anos de criação, encontra-se em processo de consolidação da infraestrutura física. Dentre os campus, a unidade Tapajós é a área que detém o maior número de projetos para a construção de prédios e laboratórios. Em toda obra de engenharia civil é de vital importância conhecer a posição do nível freático, razão pela qual se dá a necessidade de analisar o solo onde alicerces serão fixados, prevenindo assim problemas futuros com as obras. Outro fator importante é o conhecimento das variações climáticas em decorrência de precipitações na região. Na Amazônia essas variáveis são diversas, haja vista que a periodicidade das estações do ano não são regulares como em outras regiões do país. Em Santarém, o inverno amazônico é caracterizado por fortes chuvas e o verão por estiagens prolongadas.
A contribuição da geofísica na geotecnia para a investigação hidrogeológica tem como destaque o uso do método da eletrorresistividade, dada a sua aplicação indireta e eficácia em determinar os parâmetros que diferem as zonas saturadas das não saturadas.

A distribuição hidrogeológica na região de Santarém é controlada pela litologia, geomorfologia e estrutura das rochas que pertencem, principalmente, à Formação Alter do Chão (fig.1). O município apresenta exposição da Formação Alter do Chão, depositadas em ambiente continental durante o Cretáceo com rochas clásticas e espessura de $600 \mathrm{~m}$, além de sedimentos dos depósitos aluviais quaternários, que recobrem parcialmente essa formação e possuem espessura aproximada de $20 \mathrm{~m}$. A sequência litológica da Formação Alter do Chão apresenta arenitos, argilitos, conglomerados subordinados e níveis de lateritas (TANCREDI, 1996). O aquífero Alter do Chão compreende um sistema hidrogeológico com propriedades de aquífero livre e confinado e está inserido na Região Hidrográfica Dominante do Amazonas (ANA, 2005). Em dados obtidos através de inventário hidrogeológico o aquífero livre indica espessura média de $50 \mathrm{~m}$ e o aquífero confinado $430 \mathrm{~m}$, com intervalos separados por aquicludes e/ou aquitardes constituídos de estratos argilosos entre $44 \mathrm{~m} \mathrm{e}$ $80 \mathrm{~m}$ de profundidade. (TANCREDI, 1996).

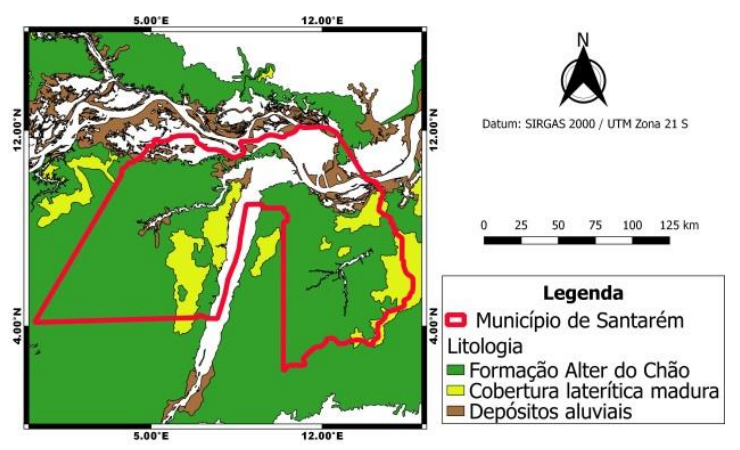

Figura 1 - Mapa Geológico de Santarém (Fonte: CPRM)

A partir do exposto, o presente trabalho teve como objetivo verificar a oscilação do nível freático do aquífero livre da Formação Alter do Chão em via não pavimentada de acesso à UFOPA - Campus Tapajós (fig.2), em 
estações distintas do ano, visando compreender o comportamento de ascensão da zona saturada e a possível relação com os alagamentos da área estudada, além de contribuir com informações que auxiliem no desenvolvimento dos projetos de infraestrutura local.

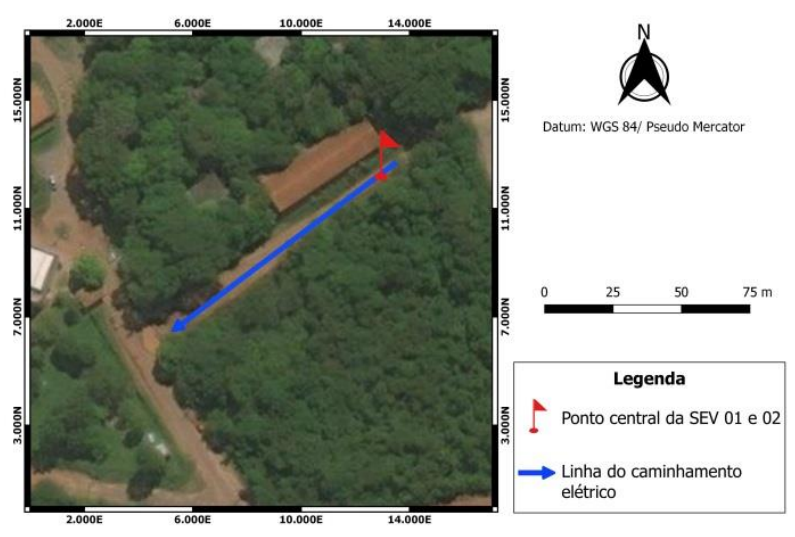

Figura 2 - Mapa de localização da área de estudo na UFOPA - Campus Tapajós (Fonte: Google Earth)

\section{Metodologia/Problema Investigado}

Nesse trabalho foram aplicadas as técnicas de Sondagem Elétrica Vertical (SEV) e Caminhamento Elétrico (CE) com o propósito de medir as variações da resistividade em profundidade, sendo a SEV pontualmente e o CE lateralmente. As técnicas consistem basicamente em injetar uma corrente elétrica no solo através de dois eletrodos ( $A$ e $B$ ) e medir a diferença de potencial em dois receptores ( $\mathrm{M}$ e $\mathrm{N}$ ), também cravados diretamente ao solo.

Em ambas as técnicas, a resistividade aparente é calculada utilizando a equação 1 (BHATTACHARYA; PATRA, 1968).

$\rho a=k \cdot \frac{\Delta V}{I}$

$\rho a=$ resistividade $\quad$ aparente $; \quad I=$ corrente $\quad$ elétrica; $\Delta V=$ diferença de potencial entre os eletrodos $\mathrm{M}$ e $\mathrm{N}$ e $k=$ fator geométrico.

O arranjo utilizado na SEV foi o Schlumberger obedecendo à relação de $M N \leq A B / 5$ (BRAGA, 2016) e o fator geométrico é dado pela equação 2 :

$k=\pi\left(a^{2}-b^{2}\right)$
No CE o arranjo utilizado foi o Dipolo-dipolo e o fator geométrico, que depende da disposição dos eletrodos ABMN na superfície do terreno, é dado pela equação 3 :

$k=2 \pi G x$

Onde, $G=\frac{1}{(1 / n)-(2 / n+1)+(1 / n+2)}$

Sendo $n=$ nível de investigação correspondente; $x=$ espaçamento dos dipolos ABMN adotados.

Foram realizadas 02 SEVs, sendo a SEV_01 em período de máxima estiagem e a SEV_02 no período de intensa precipitação. A profundidade teórica de investigação estabelecida foi de $50 \mathrm{~m}$. O CE foi realizado com a finalidade de verificar lateralmente a variação da resistividade em subsuperfície, com a profundidade teórica de investigação de $8,5 \mathrm{~m}$. A partir dos dados coletados os modelos geoelétricos foram concebidos pelo processamento dos dados de campo através do software IPI2WIN e RES2DINV e associados com a geologia e hidrogeologia locais através de informações dos perfis dos poços RIMAS (fig.3) e mapa geológico da CPRM (fig.1).

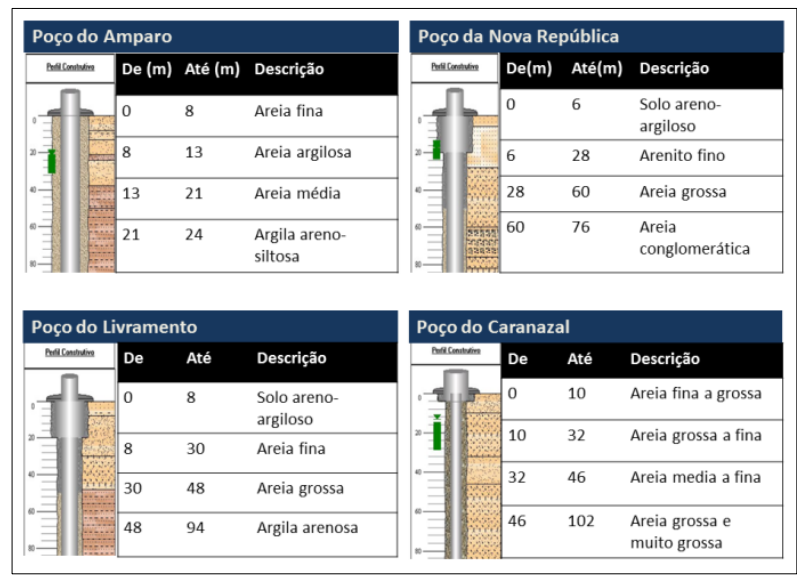

Figura 3 - Perfil dos Poços RIMAS cadastrados na área urbana de Santarém (Adaptado: CPRM) 


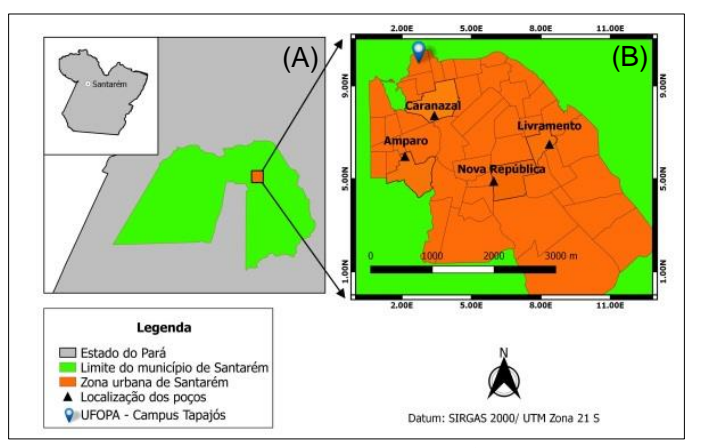

Figura 4 - (A) Mapa do município de Santarém; (B) Mapa da área urbana de Santarém e localização dos poços RIMAS.

\section{Resultados e Discussões}

Na SEV_01, realizada em 01 de dezembro de 2017, o modelo geoelétrico interpretado foi dividido em 04 camadas, a cobertura pedológica apresenta três níveis geoelétricos distintos, que compreende da superfície até $15 \mathrm{~m}$. O primeiro nível, com profundidade de $0,52 \mathrm{~m} \mathrm{e}$ resistividade aparente na ordem de $1760 \Omega \mathrm{m}$, foi associado a um solo areno-argiloso compactado. O segundo nível se estende de $0,52 \mathrm{~m}$ até $3,79 \mathrm{~m}$ apresentando resistividade $2408 \Omega \mathrm{m}$ e o terceiro nível de $3,79 \mathrm{~m}$ até $15 \mathrm{~m}$, com resistividade de $5624 \Omega \mathrm{m}$. Essa ascensão abrupta do valor pode estar associada à presença de corpos altamente resistivos, não podendo ser descartada nenhuma hipótese de interpretação. No terceiro nível, a partir de $15 \mathrm{~m}$, a resistividade sofre um decaimento para $315 \Omega \mathrm{m}$, indicando o possível limiar entre a zona saturada e não saturada. Não foi possível determinar a espessura da camada, pois ela se estende para além da profundidade teórica máxima de investigação.

$\mathrm{Na}$ SEV_02, realizada em 01 de fevereiro de 2018, adotou-se 03 camadas geoelétricas. No primeiro nível a resistividade apresentou $450 \Omega \mathrm{m}$ e profundidade de 0,5 $\mathrm{m}$, a queda dessa resistividade em relação ao primeiro levantamento está associada à saturação do solo devido a presença de água proveniente das chuvas. No segundo nível, a alta resistividade se apresentou na ordem de $5500 \Omega \mathrm{m}$, com profundidade entre $0,5 \mathrm{~m}$ até $13,5 \mathrm{~m}$. No terceiro nível a resistividade sofre um decaimento para $332 \Omega \mathrm{m}$, com profundidade em 13,5 m, ou seja, com a possível zona saturada ascendendo cerca de $1,5 \mathrm{~m}$. Conforme comparativos nas figuras 5 e 6 .

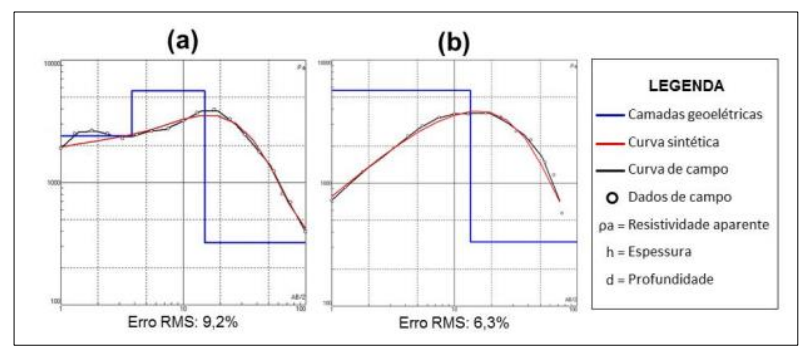

Figura 5 - (a) Plotagem SEV_01 e (b) Plotagem SEV_02

\begin{tabular}{|c|c|c|c|c|}
\hline \multicolumn{5}{|c|}{ SEV_01 - Realizada em: 01/12/2017 } \\
\hline Camada Geoelétrica & $\rho a(\Omega m)$ & $h(m)$ & $d(m)$ & Geologia \\
\hline 1 & 1760 & 0,52 & 0,52 & $\begin{array}{l}\text { Solo areno-argiloso } \\
\text { compactado }\end{array}$ \\
\hline 2 & 2408 & 3,27 & 3,79 & \multirow{2}{*}{$\begin{array}{l}\text { Solo areno-argiloso } \\
\text { compactado, com a } \\
\text { presença de corpos } \\
\text { altamente resistivos }\end{array}$} \\
\hline 3 & 5624 & 11,21 & 15 & \\
\hline 4 & 315 & - & - & Areia argilosa \\
\hline \multicolumn{5}{|c|}{ SEV_02 - Realizada em: 01/02/2018 } \\
\hline Camada Geoelétrica & $\rho a(\Omega m)$ & $h(m)$ & $d(m)$ & Geologia \\
\hline 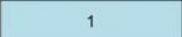 & 450 & 0,5 & 0,5 & $\begin{array}{l}\text { Solo areno-argiloso } \\
\text { compactado }\end{array}$ \\
\hline 2 & 5524 & 13 & 13,5 & $\begin{array}{l}\text { Solo areno-argiloso } \\
\text { compactado, com a } \\
\text { presença de corpos } \\
\text { altamente resistivos }\end{array}$ \\
\hline 3 & 332 & - & - & Areia argilosa \\
\hline $\begin{array}{l}\rho a(\Omega m)=\text { resistivid } \\
h(m)=\text { espessura } \\
d(m)=\text { profundidad }\end{array}$ & e aparen & & & \\
\hline
\end{tabular}

Figura 6 - Modelo geoelétrico interpretado das SEVs (01 e 02)

Em função da alta resistividade em ambos os levantamentos ( $\cong \mathbf{5 5 0 0} \mathbf{\Omega m}$ ), foi necessário a aplicação do Caminhamento Elétrico (fig.7) para mapear a subsuperfície e indicar a distribuição da resistividade lateralmente, identificando as possíveis causas da anomalia.

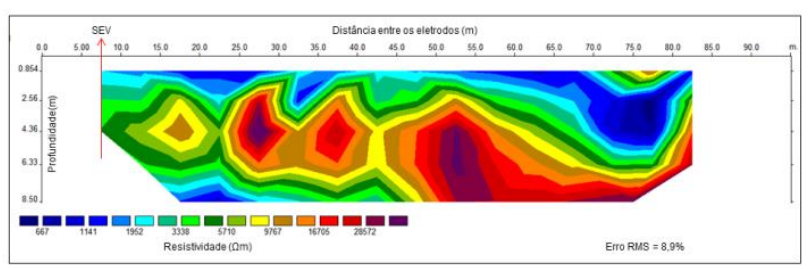

Figura 7 - Perfil de resistividade modelada

A partir dos dados do caminhamento foi possível observar que a resistividade aparente sofre uma grande variação lateral. O levantamento identificou a presença de três corpos distintos altamente resistivos. O primeiro deles está entre a posição 10 até 20 , na profundidade de $4,36 \mathrm{~m}$ e apresenta resistividade na ordem de $9760 \Omega \mathrm{m}$. O segundo corpo está localizado entre a posição 25 até 40, também numa profundidade de 4,36 $\mathrm{m}$ e apresenta resistividade na ordem de $28570 \Omega \mathrm{m}$. O último corpo está localizado entre a posição que vai de 45 até para além da 
linha de investigação e está a uma profundidade de 6,33 $\mathrm{m}$, apresentando uma resistividade da ordem de 28570 $\Omega \mathrm{m}$. Isso corrobora com as sondagens realizadas no local que indicam uma resistividade elevada tanto no período de estiagem quanto no período de chuva. Não foi possível determinar que tipos de corpos estão gerando a anomalia resistiva, não sendo descartada nenhuma hipótese. Estas podem variar desde presença de matacões até artefatos arqueológicos, haja vista que o local onde foi realizado o levantamento trata-se de um sítio arqueológico, o Sítio Porto (SCHAAN, 2015). A identificação desses corpos não será alvo do presente estudo, pois como o objetivo da pesquisa visa investigar a variação do lençol freático no aquífero Alter do Chão, os dados anômalos encontrados podem ser utilizados para o desenvolvimento de outros trabalhos mais específicos.

\section{Conclusões}

O modelo geoelétrico interpretado a partir da inversão dos dados coletados nos dois levantamentos (SEV_01 e SEV_02) mostrou a presença de camadas geoelétricas distintas, as quais foram associadas à geologia local a partir dos perfis dos poços RIMAS perfurados na região e disponibilizados pelo portal da CPRM (fig.3).

Em dezembro de 2017, a zona saturada estava a $15 \mathrm{~m}$ de profundidade e em fevereiro de 2018 variou para uma ordem de 13,5 m, indicando a ascensão em 1,5 m. A oscilação da altura do nível freático na área em estudo pode estar associada às precipitações ocorridas no período de monitoramento. O CE identificou a presença de fortes anomalias resistivas em subsuperfície que ajudaram a compreender melhor o contexto pedológico e geológico da área de estudo, bem como serviu para direcionar um novo viés para o projeto de pesquisa que irá buscar identificar a fonte das anomalias resistivas.

Para que a pesquisa possa correlacionar o comportamento de ascensão do nível freático aos alagamentos da área de estudo e consequentemente auxiliar no desenvolvimento dos projetos de infraestrutura local de maneira mais efetiva é necessário o monitoramento periódico do comportamento da dinâmica de variação da zona saturada durante um ciclo anual hidrológico, trabalho este que já está em desenvolvimento e será apresentado com maior robustez no $16^{\circ}$ Congresso Internacional de Geofísica da SBGf.

\section{Agradecimentos}

À Universidade Federal do Oeste do Pará e ao corpo docente da instituição, à SBGf pela oportunidade de divulgação das pesquisas desenvolvidas, aos laboratórios LAMGEO, LIGEO e PET-IEG que disponibilizaram seus espaços e equipamentos para 0 progresso do trabalho e ao orientador por ceder o Resistivímetro RD-1000A permitindo assim que o estudo fosse desenvolvido.

\section{Referências}

ANA. Disponibilidade e Demandas de Recursos Hídricos no Brasil. Brasília: ANA - Agência Nacional de Águas. 2005a. Cadernos de Recursos Hídricos. Disponível em: <http://www.ana.gov.br/pnrh_novo/documentos/01\%20Di sponibilidade\%20e\%20Demandas/VF\%20Disponibilidade Demanda.pdf >. Acesso em: 14 de março de 2018.

BHATTACHARYA, P. K.; PATRA, H. P. Direct current geoelectric sounding. New York: Elsevier Publishing Company, p. 134, 1986.

BRAGA, A. C. O. Geofísica aplicada: Métodos geoelétricos em hidrogeologia. São Paulo: Oficina de Textos, p. 81, 2016.

SCHAAN, D. P. e ALVES, D. T. Um porto, muitas histórias: Arqueologia em Santarém - Gráfica Supercores. Belém, 2015.

TANCREDI, A. C. F. N. S. Recursos hídricos subterrâneos de Santarém: Fundamentos para uso e proteção. 153f. Tese (Doutorado) - Centro de Geociências, Universidade Federal do Pará, Belém, 1996. 